\title{
Les rapports de Mgr L.-P.-A. Langevin avec les groupes ethniques minoritaires et leurs répercussions sur le statut de la langue française au Manitoba, 1895-1916
}

\section{Gilbert-Louis Comeault}

Volume 42, 1975

URI : https://id.erudit.org/iderudit/1007237ar

DOI : https://doi.org/10.7202/1007237ar

Aller au sommaire du numéro

Éditeur(s)

Les Éditions Historia Ecclesiæ Catholicæ Canadensis Inc.

ISSN

0318-6172 (imprimé)

1927-7067 (numérique)

Découvrir la revue

Citer cet article

Comeault, G.-L. (1975). Les rapports de Mgr L.-P.-A. Langevin avec les groupes ethniques minoritaires et leurs répercussions sur le statut de la langue française au Manitoba, 1895-1916. Sessions d'étude - Société canadienne d'histoire de l'Église catholique, 42, 65-85. https://doi.org/10.7202/1007237ar

Tous droits réservés ㄷ Les Éditions Historia Ecclesiæ Catholicæ Canadensis Inc., 1976
Ce document est protégé par la loi sur le droit d'auteur. L'utilisation des services d'Érudit (y compris la reproduction) est assujettie à sa politique d'utilisation que vous pouvez consulter en ligne. 


\section{Les rapports de Mgr L.-P.-A. Langevin avec les groupes ethniques minoritaires et leurs répercussions sur le statut de la langue française au Manitoba, 1895-1916}

Le père Louis-Philippe-Adélard Langevin venait d'être nommé vicaire supérieur des Oblats de l'Ouest et n'était pas sitôt arrivé à St-Boniface qu'il se rendait compte que les Franco-Manitobains étaient dans une situation bien précaire. Minoritaires, ils pouvaient difficilement faire valoir leurs droits.

C'est pourquoi il entreprit immédiatement une campagne de recrutement de colons venant soit du Québec soit de la France. Dès qu'il fut nommé archevêque de St-Boniface, il envoya l'abbé Jean Gaire en France pour recruter des colons. Les résultats de ses efforts ne furent pas très encourageants si bien qu'un haut fonctionnaire de l'immigration lui répondait qu'il regrettait de devoir lui dire que le nombre d'immigrants de France vers l'Ouest canadien diminuait d'année en année depuis $1893^{1}$.

$\mathrm{M}^{\mathrm{gr}}$ Langevin ne renonça pas. Il voyait des immigrants affluer au Manitoba en 1896 et réclamait avec insistance une venue en masse de colons du Québec "pour mieux résister et rendre la position à jamais imprenable dans les vallées de la Rivière Rouge et de l'Assiniboine » ${ }^{2}$. Mais le père Moïse Blais et l'abbé Joseph David Filion chargés de recrutement dans le Québec rencontrèrent une vive opposition au point que le père Blais pouvait dire : "Dans la Province de Québec on annonce peu le Manitoba et le Nord-Ouest, on les dénonce trop souvent $\gg{ }^{3}$.

Et c'est un Langevin bien peiné qui disait : "J'ai l'âme navrée quand je songe qu'un si grand nombre de mes compatriotes comprennent si peu les immenses avantages qu'offrent le Manitoba et le

1 Archives de l'Archevêché de Saint-Boniface (désormais AASB) Thomas Gilley à Mgr Langevin, le 1er janvieer 1900 .

2 Le Manitoba, le 30 novembre 1900.

3 Les Cloches de Saint-Boniface (désormais LCBS) 2, (1903), 185. 
Nord-Ouest à tous ceux qui veulent établir une nombreuse famille ${ }^{4}$. L'archevêque n'en était pas moins déterminé à tout faire pour implanter de manière durable l'Église catholique dans l'Ouest canadien. Car "fonder une paroisse », déclarait-il, "c'est l'œuvre des œuvres parce que c'est fortifier l'élément catholique et assurer le triomphe de la liberté ${ }^{5}$. Il en vint à rêver d'un véritable empire catholique dans l'Ouest, ce qui aurait ravi les Bourget et les Laflèche. L'Église y aurait le pas sur le gouvernement civil et aurait tout à dire quand il s'agirait de morale et de politique. Elle aurait mission de faire proclamer certaines vérités. L'idéal serait que cet Empire ne tolérerait aucune concession au libéralisme. Aucune alliance, aucun compromis, aucune conciliation ne serait possible. Car il lui semblait que la société laïque naissante n'était rien moins qu'une nouvelle barbarie qui serait encore cette fois-ci repoussée par l'Église, ce dernier rempart d'un ordre social aux abois.

Mais les recrues manquaient. En 1901, il n'y avait que 35,672 Catholiques au Manitoba alors qu'il y avait 44,922 Anglicans, 149,926 Méthodistes, 65,348 Presbytériens ${ }^{6}$. Cependant, la venue de nombreux immigrants de l'Europe centrale, Ruthènes, Polonais et Allemands lui fit espérer que son idéal pourrait se réaliser. Qu'une population catholique prendrait enfin le dessus sur une population protestante résolument laïque. Les Ruthènes, vu leur grand nombre, seraient un atout considérable. Les Polonais, une des races les plus brillantes d'Europe, seraient d'un grand apport intellectuel; et les Allemands, très portés pour les sciences, et par le passé, maîtres en organisation d'entreprises sociales, pourraient être d'un grand secours pour assurer la cohésion de ces groupes. Quant aux Irlandais catholiques, ils assumeraient la liaison entre les protestants et les minorités catholiques non francophones. Et quant aux Canadiens français, si longtemps habitués aux luttes religieuses et constitutionnelles, ils étaient le groupe le mieux organisé et le plus cohérent et pourraient être d'un grand secours en tout cela ${ }^{?}$.

4 Mgr Langevin à Hermas Langevin, le 15 avril 1899, cité dans A.-G. Morice, Vie de $M^{\text {or }}$ Langevin, (Saint-Boniface, Chez l'Auteur, 1919), 181.

5 Cité dans Joseph Magnan, Mor Adélard Langevin, (Winnipeg, L'Ami du Foyer, 1958), 31.

6 Census of Canada, 1901, 146-147.

7 Cette ligne de conduite a été préconisée par la Fédération des Catholiques Manitobains; son plan d'action fut élaboré par Théophile Hudon dans un pamphlet publé en 1912 . 
Les Cloches de St-Boniface exprimèrent très bien les espoirs que ces nouveaux venus faisaient naître : "Ces populations ne sont certes pas les moins intéressantes et leur développement incessant sera peut-être un jour une des grandes forces de l'Église catholique au Manitoba »8. Langevin ajoutait:

Ces braves populations... sont assez intelligentes pour venir s'emparer des belles terres du Manitoba dédaignées par tant de Canadiens-français qui préfèrent aller s'étioler dans les manufactures des Etats-Unis que de vivre libres et indépendants sur le sol vierge de leur immense et si beau pays ${ }^{9}$.

Il restait à voir si les Polonais, les Allemands catholiques, et surtout les Ruthènes, seraient d'accord avec les vues de Langevin.

Les Ruthènes étaient pour l'archevêque le cas le plus complexe. A cet effet un curé disait que les plus grands ennemis de ces pauvres gens n'étaient pas les gens qui leur étaient des étrangers mais leurs propres gens qui les harcelaient avec toujours de nouvelles armes. Il ajoutait, cependant, que pour compenser, les Polonais repoussaient courageusement ces insinueuses attaques, étant plus instruits et plus religieux ${ }^{10}$. Mais Langevin ne désespérait pas car il était convaincu que les Ruthènes adhéraient à la foi catholique :

Faut-il le redire encore? Une faible partie des Ruthènes appartient à la religion dite grecque orthodoxe, tandis que la grande majorité professe la religion catholique romaine. Bien que suivant un rite différent du rite latin, les Ruthènes catholiques sont sous la juridiction du Pape de Rome et des Évêques en communion avec lui ${ }^{11}$.

À ces gens, il rappelait que l'Église catholique n'était infaillible que sous le rapport de l'intégrité de la foi et pouvait par ailleurs s'adapter aux besoins de chaque nation. Il les assurait qu'en lui portant respect ils reconnaissaient que le Pape était leur guide spirituel. Il leur recommandait de se méfier de leurs faux évêques et de leurs faux prêtres qui semaient la rumeur que la hiérarchie latine cherchait à faire disparaître le rite ruthène.

Pour s'assurer leur adhésion encore davantage, il se déclara gardien et défenseur de leur rite. En 1904 , il prêtait $\$ 30,000$ aux

8 LCSB, 3, (1904), 317.

9 Idem..

10 The North West Review, le 6 février 1901.

11 LCSB, 9, (1910), 3. 
Basiliens ${ }^{12}$ pour la construction d'une église où l'on pourrait desservir environ 5,000 Ruthènes de Winnipeg. Cela pour couper l'herbe sous le pied des Presbytériens qui, disait-on, allaient construire une église de $\$ 40,000$ aux environs. On avait de même construit l'église de Holy Ghost à Winnipeg ${ }^{13}$ et on consacrerait plus tard d'importantes sommes d'argent à la construction d'édifices religieux, couvents, hôpitaux, presbytères, à l'achat des terrains voulus et à subventionner un journal ruthène. Langevin rappelait avec insistarce ce qui était fait en faveur de ses Ruthènes. Même le clergé du Québec s'intéressait à eux. Aussi les Pères du Premier Concile de Québec avaient bien voulu donner une preuve de l'intérêt qu'ils portaient aux Ruthènes en promettant de donner, shaque année, pendant dix ans, pour les œuvres ruthènes, quatre piastres par mille diocésains ou de faire une quête qui donnera les mêmes résultats. En retour les Évêques de la Province ecclésiastique de St-Boniface consentaient à renoncer pendant dix ans à la quête des écoles du Nord-Ouest ${ }^{14}$.

Mais l'aide financière n'était pas tout. Il fallait surtout des prêtres pour maintenir ces gens-là dans le giron de l'Église. Le $1^{\text {er }}$ mai 1897, la Sacrée Congrégation de la Propagande de l'Église Catholique Romaine avait mis les prêtres de l'Église catholique grecque sous la juridiction des évêques catholiques romains de l'Amérique du Nord. Déjà, auparavant, cette Congrégation avait décidé qu'aucun prêtre marié ne devait venir en Amérique. Dès 1896, Langevin avait demandé aux Oblats qui parlaient allemand ou polonais de faire $\mathrm{du}$ ministère dans les paroisses ou missions ruthènes. En 1898, il demandait au Métropolite catholique grec de Galicie, le comte André Sheptycky, d'envoyer un clergé célibataire ruthène au Canada. Mais ce fut un Rédemptoriste belge qui vint cette année-là : le père Achille Delære. D'autres Rédemptoristes suivirent bientôt. Enfin les premiers prêtres ruthènes apparurent quatre ans plus tard. Ils ne semblent pas avoir plu à Langevin qui écrivait au Cardinal Gotti, le préfet de la Sacrée Congrégation de la Propagande, dans les termes suivants: «Les passages des prêtres séculiers ruthènes au sein de nos colonies ont fait plus de mal que de bien et ceux qui sont véritablement

12 Les Basiliens étaient considérés comme l'élite du clergé ruthène.

13 AASB, Rapport de la Commission chargée par le Premier Concile du Canada d'étudier la question des Ruthènes et de soumettre quelques conclusions, le 28 septembre 1909.

14 Circulaire au Clergé de l'Archevêché de Saint-Boniface, le 2 février 1910, Mandements des Évêques du Nord-Ouest, 3, (1905-1915), 285. 
religieux parmi ces peuples s'attachent seulement aux prêtres latins $\gg^{15}$. Vers la fin de l'année 1904, il croyait avoir trouvé la solution. Pour leur fournir des prêtres qui pourraient célébrer dans leur rite, former des prêtres canadiens à cette fin et, avec l'aide des Rédemptoristes, constituer un clergé ruthène. Après de longues négociations avec Rome, le père Delære passa du rite latin au rite ruthène. D'autres Rédemptoristes firent de même et bientôt ce fut les abbés Adonias Sabourin, Désiré Claveloux, Joseph Gagnon, Joseph Jean et Arthur Desmarais qui firent de même. Il lui semblait que c'était là le meilleur moyen d'empêcher les hérésies, les erreurs et d'empêcher les doctrines protestantes de s'implanter parmi eux. Ce serait un excellent moyen de prévenir la venue des prêtres mariés dont les épouses, déguisćcs en bonnes ménagères, n'auraient d'autres soucis que de s'assurer un revenu intéressant.

Si l'archevêque avait espéré que les Basiliens seraient d'un grand secours pour la conservation de la foi chez les Ruthènes, il fut plutôt désappointé. D'abord les Ruthènes se méfiaient de ces "monkish advisors " qui allèrent jusqu'à conseiller la soumission aux évêques latins et la cession des biens de leur église aux diocèses de ces évêques français dont on disait qu'ils s'efforçaient de latiniser leur rite et qui par conséquent n'étaient rien moins que des loups couverts d'une peau de mouton ${ }^{16}$. Il y avait aussi un journal américain ruthène, le Svoboda, qui encourageait fortement les Ruthènes du Manitoba de se joindre à l'Association des Paroisses Ruthènes des États-Unis d'Amérique pour ainsi s'affranchir de la hiérarchie latine. Aussi quelques paroissiens de Saint-Vladimir abandonnèrent cette paroisse pour fonder une Église grecque catholique indépendante.

Bien divisés déjà, un nouvel élément de discorde devait s'ajouter à tous les autres. En septembre 1903, un individu nommé Séraphim fit son apparition au Manitoba et se disait patriarche de l'Église orthodoxe de toutes les Russies. Des dignitaires ecclésiastiques catholiques prétendaient qu'il n'aimait rien d'autre que l'argent et la boisson et qu'il ordonnerait à la prêtrise, n'importe qui, pour la somme de $\$ 50.00$ sauf les cultivateurs qu'il ordonnerait gratis. Il obtint au départ beaucoup de succès mąis ce fut un feu de paille. Des crises d'aliénation mentale le portèrent à construire une cathé-

15 AASB, Mgr Langevin au Cardinal Gotti, le 11 août 1902.

16 Michæl H. Marunchak, The U'krainian Canadians : A History, (Winnipeg: Ukrainian Free Academy of Sciences, 1970), 89. 
drale de ferraille à Winnipeg. Les prêtres qu'il avait ordonnés l'abandonnèrent et grâce à des subventions venant des Presbytériens formèrent une Église grecque indépendante. Langevin s'éleva contre tout cela et refuta avec indignation les mensonges tels que celui-ci :

Do not trust the French clergy, because they are your enemies, they try to deprive you of the treasure of your rite. Do not trust the Archbishop of St. Boniface, nor the missionaries he sends you. Do not place your church lands, nor your churches under his authority, because he will oblige you to pay the tithes, that is the tenth part of everything you possess, the tenth cow, the tenth chicken, the tenth goose, the tenth turkey... 17

Langevin s'était bientôt rendu compte que la lutte pour gagner ces âmes nécessiterait une épreuve de force qui pourrait facilement dégénérer en conflit politique. Les protestants persistaient de même. Eux aussi voyaient là un champ propice à l'établissement de leur religion, mais l'archevêque voyait leur prosélytisme d'un bien mauvais œil. C'était en vue de ce prosélytisme protestant que l'on construisait des hôpitaux, que l'on distribuait des médicaments, des vêtements, que l'on faisait main basse sur les écoles modèles, que l'on préconisait le socialisme, que l'on insinuait que les Canadiens français étaient ici ce que les Polonais avaient été en Galicie. Effectivement Langevin ne pouvait que condamner le Radok, journal d'inspiration presbytérienne et subventionné par le parti libéral du Manitoba et le Prairie Farmer, aussi d'inspiration presbytérienne et pendant un certain temps propriété de Clifford Sifton. En contrepartie il recommandait à tous les catholiques du Canada « de prendre en main, hardiment et vigoureusement, la cause de l'Église Ruthène dans l'Ouest ». Si non « une immigration se tournera contre eux (et) ils auront à faire face à un nouvel ennemi ${ }^{18}$. Mais alors que faudra-t-il faire?

On cherche à me convaincre qu'avec de la tolérance on gagne plus qu'avec trop de chaleur dans la défense de nos intérêts... mais lorsqu'un berger voit venir le loup, va-t-il se contenter de l'attendre à la porte et de lui dire bien poliment : 'S'il vous plaît, monsieur le loup, veuillez donc avoir la bonté de ne pas entrer dans la bergerie ?' Non, il prend sa houlette

17 AASB, Mgr Langevin 'To the Catholics of the Ruthenian Rite in Winnipeg and in the Colonies of the Diocese', s.d.

18 LCSB, 8, (1909), 91. 
et chasse avec toute promptitude et l'ardeur possible. Quand le salut des âmes est en jeu, une âme épiscopale sait voir audelà des indifférences de rite et de race 19.

A la grande consternation de l'archevêque cependant, les protestants ne furent pas le moins du monde déconcertés et ne cessèrent de dénoncer les menées de Langevin c'est-à-dire sa politique de «latinization » et de "frenchification », deux termes popularisés par le Free Press. Aussi, la première difficulté que l'archevêque avait rencontrée en toute cette affaire des Ruthènes c'était l'inscription des biens ecclésiastiques au nom de la Corporation Archiépiscopale plutôt qu'à une commission de paroissiens indépendants du curé. Il considérait que ce mode de tenure était d'inspiration protestante et même avait un relent de gallicanisme, et il voulait s'assurer que des hérétiques, des schismatiques ou des protestants ne puissent s'accaparer des églises qui avaient été construites à ses frais ${ }^{20}$. En 1913, Joseph Bernier, représentant de St-Boniface à l'Assemblée législative du Manitoba, avait soumis un projet de loi stipulant que l'Église grecque ruthène devait assigner toutes ses propriétés à la Corporation Archiépiscopale. Mais le journal Ukrainian Voice fut indigné. Il voyait Langevin, qui se posait en sauveur, comme n'étant rien autre qu'un vil profiteur convoitant les quelques sous gagnés péniblement par les Ruthènes et ajoutait : «If the foundations of all Catholicism rests on the incorporation of property, and in general on property, then the Catholic Church is a business corporation or a company, like the C.P.R. ... We shall tell the French : 'Stop tampering with us and go and redeem your France which is perishing' 22 .

D'autre part, le Svoboda continua ce genre de polémique et, en 1910, s'ajoutait celle du consul d'Autriche-Hongrie, à Winnipeg, qui s'était joint aux récriminants. Selon ce dernier, les prêtres canadiensfrançais n'avaient rien d'autre en vue que de se servir des Ruthènes pour se créer un empire :

What the Ruthenians chiefly object to is that the French bishop wants to force French priests upon them... the Archbishop is sending a lot of French clergymen over to Aus-

19 A.-G. MORICE, op. cit., 279.

20 Circulaire au Clergé, le 15 août 1910, Mandements des Evêques de Saint-Boniface, 3, (1905-1915), 313.

21 Paul YUzYK, The Ukrainian Greek Orthodox Church of Canada, 1918-1951, University of Minnesota, (Thèse de doctorat, 1958), 109. 
tria to study the Ruthenian language and to go over to the Ruthenian church, so as to be able to return here and take up parishes among our Ruthenians. It seems as if it were a determined purpose not to allow the business to go out of French hands... The chief point seems to me to be, not the question of the difference of rite, but the question of French domination in the Catholic church. I can assure anybody, however, who is working in that direction, that he is working at a hopeless task, and that our people, who are coming in this country, are of different stock than the halfbreeds of Louis Riel 22.

Les rêves de l'évêque catholique n'étaient plus possibles et on s'en rendit bien compte après l'expérience de Sifton. En 1906, les Basiliens avaient acquis des terrains dans ce district, en vue d'assurer des services religieux aux Ruthènes des environs. Faillite complète. Le père Filas pouvait écrire aux pères Dydyk et Kryjanowski : "Secouez la poussière de vos chaussures et repliez-vous sur Winnipeg, où vous serez mieux reçus » ${ }^{23}$. Mais Langevin n'était pas homme à abandonner la partie si facilement et il envoya à Sifton l'abbé Adonias Sabourin qui venait de passer du rite latin au rite ruthène, avec mission d'établir une place forte catholique en ces lieux. Mais sitôt rendu là, l'abbé dut se rendre compte que ce centre

always had the leading step in religious disturbances among Ruthenians with the Presbyterians sending $\$ 10,000$ a few years ago to erect a mission named the 'Hospital' placed under the direction of the 'ill-famed' Dr. Reid. The shameless imposter Séraphim has performed one of its most numerous ordination in this town and Apostate priests are trying to take control of the Catholic Church... A dual is going on between the Orthodox and the Catholic faith. Unhappily, schismatics have the upper hand, so far, because they are left in quiet possession of the Catholic Church built by Catholics for Catholic worship, owing to a flaw in the contract that was made in the purchase of the land from the C.N.R. ${ }^{24}$.

22 Manitoba Free Press (désormais MFP) le 30 août, 1910.

23 Émilien Tremblay, Le Père Delare et l'Église Ukrainienne du Canada, (Press of the Catholic Ukrainian Bishop of Saskatchewan, 1960), 32.

24 AASB, Fonds Sabourin, J.-Ad. Sabourin à A.E. Burke, le 29 septembre, 1909. 
Cependant, Sabourin n'admettait pas que les Ruthènes cherchent à assurer leur salut sans le secours de Rome, ni quoi qu'en pense le ministre méthodiste J.S. Woodsworth, sans le secours de Dieu ${ }^{25}$. Selon lui, il ne devait pas $\mathrm{y}$ avoir de prêtres ruthènes paresseux dans son district et il insistait pour que les propriétés ecclésiastiques soient enregistrées au nom de la Corporation Archiépiscopale ${ }^{26}$. Va sans dire que tout cela restait sans effet. Aussi, dès 1911, Langevin devait lui écrire : "Que faire au milieu d'un peuple dont on n'a pas la confiance? Inutile de se dissuader, tu n'as pas assez la confiance des Ruthènes à Sifton où ils sont trop travaillés par les schismatiques et les protestants $\gg 27$.

Dès 1907, l'évêque Ortynski, premier évêque ruthène aux ÉtatsUnis, écrivait au père Delære que ce que se proposait Langevin, de faire passer des prêtres du rite latin au rite ruthène, serait une faillite complète. Les Protestants et les schismatiques, selon ce dernier, allaient être les seuls à profiter de cet état de choses ${ }^{28}$. Comme il fallait s'y attendre, les Basiliens prétendirent aussitôt que la nomination d'un évêque ruthène qui viendrait, accompagné d'un clergé ruthène véritable, serait la seule planche de salut. De plus, A.E. Burke, président de la Church Extension Society qui avait fourni des fonds à Langevin pour l'œuvre ruthène, commença à se demander si la manière de faire de l'archevêque était efficace :

I am sorry to say that there does not appear to be any good ground for hope of much success from the Latin end of that Ruthenian question as the Ruthenians are beginning to refuse money from Latin sources. There does not appear to be any other solution to this question than the one which everybody is getting more convinced of every day - the appointment of a Ruthenian bishop, and he will save them all to the Church. In any other hypotheses, it seems a waste of time, energy and money 29.

Sabourin avait lui-même caressé cette idée mais voulait retarder l'échéance «car il n'est pas du tout impossible », avouait-il,

25 Idem.

26 AASB, Fonds Sabourin, Joseph Gagnon à J.-Ad. Sabourin, le 4 août 1911.

27 AASB, Mgr Langevin à J.-Ad. Sabourin, le 16 mars 1911.

28 Émilien Tremblay, op. cit., 136.

29 AASB, Fonds Sabourin, A.E. Burke à J.-Ad. Sabourin, le 11 janvier 1910 . 
"que la nomination d'un évêque ruthène ne transplante ici un clergé dont l'esprit est fort différent du clergé canadien 》 ${ }^{30}$. Langevin aussi s'opposait à la nomination d'un tel évêque. Déjà, en 1900, il avait refusé un coadjuteur ruthène en son diocèse, alléguant plusieurs raisons :

Vous savez ce que je pense de la nomination d'un sous-délégué ruthène, je crois que ce sera une vraie calamité. C'est le plus sûr moyen d'éterniser le rite ruthène en Galicie. Puis au danger du schisme s'ajoutera le danger de l'influence hérétique dans ce pays protestant... Enfin avant longtemps l'on demandera un évêque du rite ruthène, déjà on en a parlé. Au contraire avec des religieux du rite latin comme les Oblats de Marie Immaculée... on laisserait la liberté de garder quelques pratiques extérieures du rite ruthène, en dehors de la messe et de l'administration des sacrements, nous réussirons à unifier ces peuples... ${ }^{31}$.

Il se demandait également comment iraient les choses avec deux évêques ayant la même juridiction, dans le même territoire, fussentils de rites différents. Cela compliquerait tout et créerait un état dans l'état. Cela faciliterait l'action des protestants auprès de ces gens en les divisant davantage, eux qui déjà s'éloignent de leur rite. En tout cas, il voulait bien le salut des Ruthènes mais « je ne veux pas me trouver embarrassé avec un évêque qui verra d'un mauvais œil les prêtres latins passés au rite ruthène et forcés d'être sous la tutelle des prêtres ruthènes ${ }^{32} \gg$. Cependant, il ne se cachait pas ce qui viendrait : un jour, il $\mathrm{y}$ aura un évêque ruthène avec pleine juridiction mais «il s'arrangera comme il voudra ». Nous n'aurons rien à faire avec ces Ruthènes... ${ }^{33}$

Le 15 juin 1912, on annonçait l'élection de Niceta Budka au poste d'évêque de tous les Ruthènes du Canada. En bien peu de temps les relations entre le nouvel élu et les prêtres canadiensfrançais qui œuvraient parmi les siens furent des plus tendues. Tout d'abord Budka reconnut le Ruthenian Canadian qui faisait campagne contre ces prêtres. Langevin cessa aussitôt de subventionner ce journal. L'évêque ruthène riposta en spécifiant qu'il fallait cesser

30 AASB, Fonds Sabourin, J.-A. Sabourin à Mgr Tiberghien, le 1er février 1910.

31 AASB, Mgr Langevin à Mgr Falconio, le 5 octobre 1900.

32 AASB, Mgr Langevin à J.-Ad. Sabourin, le 8 mai, 1909.

33 AASB, Mgr Langevin au père Delære, le 11 juin, 1909. 
tout à fait de faire passer des prêtres du rite latin au rite ruthène. Il ajoutait que Sabourin aimait mieux ses chevaux que ses $\mathrm{Ru}-$ thènes ${ }^{34}$. En retour, Sabourin se plaignit à Langevin que Budka disait à tout venant que les Ruthènes n'avaient jamais accepté les prêtres de rite latin passés à leur rite, et l'accusa d'avoir causé un schisme et de demander à Rome la permission de faire venir de Galicie des prêtres mariés.

Plutôt que de faire du ministère dans de telles conditions, quelques Rédemptoristes, les abbés Gagnon, Desmarais et Claveloux entre autres, demandèrent leur rappel et leur réintégration au rite latin. Langevin en informa Budka sans ménagements : "Ce sont les dispositions adverses de Votre Grandeur qui les ont déterminés à abandonner le rite Ruthène $\$ 35$. Sabourin aussi songea au retour : «Pour moi je ne vous le cache pas, la question de retourner au rite latin se pose très sérieusement ». Mais, tenace qu'il était, il décida de rester car "notre départ faciliterait cette introduction (de prêtres mariés) sous le faux prétexte que le peuple ruthène ne veut plus de prêtres latins, vu que ceux-ci trouvent trop difficile de desservir les Ruthènes dans leur langue et leur rite ${ }^{36}$. En fin de compte Langevin avertit Budka qu'il n'était pas d'accord avec lui sur bien des sujets et en profitait pour lui rappeler qu'il avait fait de grands sacrifices pécuniers pour venir en aide aux Ruthènes. Puis il lui lança cet avertissement: «...si votre Grandeur les rejette qu'elle en subisse les conséquences et qu'elle ne soit pas étonnée que l'œuvre des Ruthènes cesse d'avoir pour les catholiques latins, surtout pour la Province de Québec, les mêmes sympathies ardentes et religieuses $\$ 37$. De toute évidence, l'archevêque s'était rendu compte que son empire catholique n'avait été qu'un rêve irréalisable.

Mais, au départ, les immigrations massives du début du siècle n'avaient pas empêché l'archevêque de penser aux siens, les Canadiens français. Aussi, parlant de ces milliers de Galiciens qui arrivaient dans l'Ouest, il affirmait à Alexandre Guasco, secrétaire de la Sacrée Congrégation de la Propagande de la Foi, qu'il «...faut lutter pour maintenir l'influence catholique et française dans un

34 AASB, Fonds Sabourin, J.-Ad. Sabourin à Joseph Gagné, le 21 aout 1913.

35 AASB, Mgr Langevin à Mgr Niceta Budka, le 6 décembre 1913.

36 AASB, Mgr Langevin à Mgr Niceta Budka, le 23 juillet 1913.

37 Idem.. 
pays protestant et anglais ${ }^{38}$. Il fallait donc qu'il y ait un empire dans l'empire capable d'assurer une union qui serait en mesure de dénouer la question des écoles. Malheureusement, il était à cent lieues de voir les conséquences d'une telle stratégie. Mais c'était dans sa logique, car, il en était bien convaincu, "qui perd sa langue perd sa foi ». Le dicton ne pouvait mentir. Le régime d'école bilingue serait le seul qui pourrait assurer aux Galiciens l'instruction en leur langue et ainsi sauvegarder leur foi car l'anglais, au Canada, c'était « une force pour l'hérésie ${ }^{39}$.

Comme il était loin de prévoir les répercussions qui se feraient sentir à l'endroit du français dans les écoles publiques au Manitoba, si l'on faisait profiter les catholiques autres que de langue française, de la clause ayant trait au bilinguisme dans le règlement LaurierGreenway! Mais, dès l'arrivée des premiers immigrants d'Europe centrale, il se préoccupa de leur instruction et s'adressa à Rodmond Roblin, le premier ministre du Manitoba, en ces termes :

\begin{abstract}
Allow me to state before you the Galician and Polish people who have come lately from the Empire of Austria... in the great to the Roman Catholic Church of the latin or ruthenian rite, and the balance belong to the Russian Church which believe as we do in the Apostle's creed and in seven sacraments. Besides hundreds in Winnipeg and in many colonies in rural districts without any instruction whatever; and in Winnipeg alone, the Reverend Fathers of the Holy Ghost's Church, assure me that they could get, before long, about two hundred children speaking Polish or Galician, if they had the financial means to provide for school rooms and school teachers... Consequently, I take the liberty... to ask the Government to provide means to instruct and educate the children... in their own idioms 40.
\end{abstract}

Néanmoins, il en est qui pensait tout autrement. Ms voulaient plutôt des écoles publiques dans tous les endroits où se trouvaient ces groupes minoritaires, et que l'école obligatoire devienne loi car ce qui était important c'était que l'anglais soit enseigné. Langevin n'était pas d'accord et le fit bien savoir en un fameux discours qui mérite citation intégrale, car c'est là qu'il proclame que si la langue

38 AASB, Mgr Langevin à Alexandre Guasco, le 10 décembre 1903.

39 A.-G. MORICE, op. cit., 248.

40 AASB, Mgr Langevin à R.P. Roblin, le 28 janvier 1901. 
et la foi vont de pair pour les Canadiens français, il devrait en être de même pour les Galiciens :

\begin{abstract}
On... doit établir parmi eux des écoles où la langue anglaise sera enseignée selon les exigences de la loi, mais puisque cette même loi concède l'enseignement bilingue, c'est-à-dire l'enseignement d'une autre langue que l'anglais pour ceux qui ne parlent pas cette dernière, ces étrangers (les Galiciens) ont droit de faire instruire leurs enfants dans leur propre langue, et c'est là leur désir le plus ardent. Mais si tout le monde admet que l'anglais doit être enseigné dans les écoles du Manitoba, tous ne sont pas d'avis que l'on doive enseigner aussi la langue maternelle des Galiciens; quelques-uns même ont déclaré hautement qu'il vaudrait mieux n'enseigner partout que l'anglais! Prétention exorbitante, injuste et dangereuse pour la paix de notre pays ${ }^{41}$.
\end{abstract}

L'archevêque demandait également d'où venait cet intérêt soudain pour les Galiciens : "Ne serait-ce parce que la grande majorité des Galiciens appartiennent à l'Église catholique ? » À ceci il ajouta que dans l'intérêt de la paix du Canada il vaudrait mieux que chacun s'en tienne à ses propres affaires et jeta cet avertissement:

Nous entendons bien, nous de nous mêler de ce qui nous regarde. Si ceux qui menacent en ce moment les lois scolaires actuelles, parce qu'elles permettent jusqu'à un certain point, l'enseignement bilingue et l'enseignement religieux en faveur des galiciens, ils se trompent beaucoup. ${ }^{42}$.

Dans tout cela, Langevin s'était prononcé en faveur du multilinguisme au Manitoba. Il préconisera cette théorie jusqu'à sa mort en 1915. Entre temps, on discutait cette question de l'instruction des Galiciens. Le Révérend George Bryce suggérait de demander au gouvernement du Dominion, de remettre à la Province les montants accrus en intérêts, sur les sommes réalisées lors de la vente des terres réservées pour les écoles. Et ceci pour défrayer le coût de l'instruction aux nouveaux venus. Car, disait-il, il serait tout à fait impossible de mettre en œuvre des écoles galiciennes sans cet argent. L'abbé A.-A. Cherrier combattit ce projet avec ardeur pour la simple raison que les catholiques ne croyaient pas la question des écoles réglée tant que ceux de Winnipeg seraient l'objet des injustices dont ils souffraient ${ }^{43}$.

$41 \quad L C S B, 1,(1902), 8$.

42 Ibid., 11-12.

43 The North..West Review, le 25 janvier 1902. 
$\mathrm{Au}$ cours de ce débat, on s'était adressé à Robin. Ce dernier dit qu'il ne s'était jamais désintéressé de cette question mais la Province n'était pas en mesure d'assumer ce surcroit de dépense, surtout si une loi d'école obligatoire était promulguée ${ }^{44}$. Ce n'était pas tout à fait la vérité. L'élection provinciale de 1903 avait démontré à Roblin que Langevin pouvait valoir bien des votes des Canadiens français en faveur du parti conservateur. Pas surprenant qu'il ait consulté l'archevêque au sujet de l'instruction obligatoire et que ce dernier lui avait répondu que ça n'irait pas sans difficultés tant que les écoles séparées de Winnipeg ne seraient pas reconnues comme des écoles publiques ${ }^{45}$.

Il y avait sans doute un bon montant d'argent dans les coffres fédéraux provenant de la vente des terres réservées aux écoles, mais il semble bien que Wilfrid Laurier n'aurait pas consenti à le remettre au gouvernement du Manitoba, à moins que Roblin ne règle la question à la satisfaction de l'archevêque. Mais un tel règlement eut provoqué des réactions défavorables au premier ministre du Canada. Aussi Roblin préféra temporiser ce en quoi il n'avait rien à perdre, tant que les libéraux seraient au pouvoir à Ottawa. La partisanerie politique allait donc jouer un grand rôle dans l'établissement d'écoles pour ces nouveaux venus et, en effet, quelques semaines avant l'élection provinciale de 1907, Robert Fletcher, chef de bureau du département de l'Éducation, écrivait à D. Fallis, à la demande de W.B. Waddell, représentant de Minnedosa à la Législature, qui le chargerait de créer un district scolaire :

There is a special reason for prompt attention, as there is a heavy vote in that locality and it is almost essential to split the vote in order to give Mr. Waddell a chance of election. The Galician vote is usually liberal... It therefore behoves us to put the machinery in motion promptly ${ }^{46}$.

De plus, Fletcher vit à ce que les piliers du parti conservateur soient bien au fait de tout ce que le gouvernement Roblin avait accompli en faveur des immigrants de l'Europe centrale. Aussi, écrivait-il à

44 Canadian Annual Review, 1902, 467.

45 AASB, Mgr Langevin à R.P. Roblin, le 5 février 1906.

46 Archives provinciales du Manitoba (désormais APM) Education Chief Letter Book, 1905-1911, Robert Fletcher à A.B. Fallis, le 20 février 1907. 
Glen Campbell, qui avait représenté Gilbert Plains à la Législature, comme suit :

Since the present government came into power, we have established sixty-nine schools that are purely Galician or practically so. We have a Ruthenian Training School at Brandon which was opened on January 1st 1908 with a class of fortythree students. In January 1909 we opened a Polish Training School at Winnipeg with a class of eleven students and we have recently transferred eight Polish students from the Brandon school to Winnipeg. While we operated the one school we endeavored to have both Ruthenian and Polish students. There are now seventeen in the Polish Training School and between twenty-five or 'thirty in the Ruthenian Training School. We established our first training school in February 1905 and closed it on July 1st, 1907... From that school we graduated twenty nine students as teachers for the Galician schools 47 .

Et pendant que le gouvernement du Manitoba établissait des écoles bilingues dans des districts habités par des «étrangers» en informant ceux-ci de tout ce qu'il faisait pour leur trouver des instituteurs, et cela pour des objectifs politiques, Langevin ne cessa de réclamer des dictricts scolaires pour les Galiciens et des instituteurs pour les conserver à la religion catholique. Également, il proclamait de vive voix que la langue d'instruction devait être leur langue maternelle. C'était la conséquence logique du système bilingue reconnu par la loi!

Entretemps, John W. Dafœ, rédacteur du Free Press, était revenu de son enthousiasme au sujet de l'immigration des «centraux 》. Avant 1907, il croyait que c'était nécessaire et même bienfaisant pour l'Ouest, mais dorénavant, il croirait que ça allait être au détriment de la continuité anglo-saxonne. Un fort courant d'opinion se manifestait en faveur de « a new and significant race known as Canadians ». Et le rédacteur du Free Press reprochait aux Polonais, aux Ruthènes et aux Allemands, de s'obstiner à conserver leur identité. Il s'en suivit que, chez les Anglo-Saxons, on fut de plus en plus d'avis que ces minorités devraient être tout simplement assimilées, de gré ou de force. Ils se sentaient menacés et en avaient assez de ces expériences multiculturelles et multilinguistiques. Toutes ces

47 APM, Education Chief Letter Book, 1905-1911, Robert Fletcher à Glen Campbell, le 5 janvier 1910. 
minorités devraient être mises dans le même creuset anglo-saxon, le «melting pot », et le régime d'instruction publique devrait à l'avenir tendre à leur faciliter l'accession à la nouvelle société.

Dafœ était gien déterminé à canadianiser tous les immigrants, et, à cette fin, il s'objectait à la clause de l'Acte des Écoles du Manitoba permettant le bilinguisme; et de plus, il préconisait l'instruction obligatoire. Une confrontation avec l'archevêque de StBoniface devenait dès lors inévitable. Quand, en 1908, un projet de loi qui allait rendre l'instruction obligatoire pour l'école primaire fut discuté en chambre, Dafæ écrivait: «since church facilities for education were inadequate the Archbishop preferred ignorance to secular education $\gg 48$. Les Cloches de Saint-Boniface retorquèrent que «purely secular education bred ignorance like a swamb bree flies » ${ }^{49}$. Dafœ y alla d'un éditorial : «in a country like ours where so many nationalities are settling in our midst, it is imperative that the children of these different nationalities should be taught the same ideals of citizenship as our natural born Canadian $>50$.

Toutefois, Langevin ne cessait de recommander aux ruthènes et aux Catholiques polonais et allemands de réclamer des instituteurs bilingues, qui assureraient la conservation de leur langue à leurs enfants et, par là même, la conservation de leur foi. De plus, il continua de s'opposer à la campagne du Free Press et du parti libéral contre le bilinguisme et contre tous les «centraux» devenus un danger pour l'État, il rappelait aux parents leurs responsabilités envers Dieu, et leur obligation d'assurer à leurs enfants, la connaissance de leur langue maternelle, gardienne de leur foi. À ceci, il ajoutait qu'il était de leur devoir de prendre la défense de leur archevêque et même de s'attaquer à ses ennemis ${ }^{51}$. Mais il était loin de penser que, étant archevêque de Saint-Boniface il était considéré par les Anglo-Saxons comme une autorité chez les Canadiens français et, que le fait d'invoquer la clause du bilinguisme en faveur de toutes ces minorités, compromettait la cause du français, pour bien des générations à venir, car les adversaires des écoles bilingues, advenant leur accession au pouvoir, en profiteraient pour régler la question globalement.

48 Murray Donelly, Dafee of the Free Press, (Toronto, Macmillan of Canada, 1968), 57.

49 Idem.

50 Idem.

$51 \quad L C S B, 10,(1911), 52-53$. 
En 1914, le Free Press s'inquiétait véritablement de cette population slave au Manitoba, entre 50 et 60 mille, la plupart Ruthènes ou Polonais venant de la Galicie ${ }^{52}$. En juillet de cette annéelà, ce quotidien de Winnipeg avertissait ses lecteurs pour la centième fois des dangers que pouvait comporter la présence de tous ces étrangers, tous imbus d'idéals nationaux divergents et qui feraient du Canada un pays multilingue. De plus, il y avait danger qu'un mouvement national-clérical chez les Ruthènes tende à rien moins qu'à établir, dans l'Ouest canadien, une nationalité ruthène distincte qui aurait son langage, ses institutions, ses costumes et ses idéals formant ainsi un état dans l'état. Qui étaient les grands coupables ? Le gouvernement Roblin qui encourageait et même subventionnait tout cela pour obtenir le suffrage de ces gens et aussi la hiérarchie catholique sous l'impulsion de l'archevêque de St-Boniface. Et au sujet de celui-ci, le Free Press disait :

Archbishop Langevin's encouragement of the Ruthenian national movement is in keeping with his well-defined religiopolitical programmes. His idea is to create conditions in the Western Provinces which will enable him to bring various Governments to their knees and compel them to yield him concessions upon demand. A compact Ruthenian organization, animated by race feelings, and subject, in large measure, to clerical control would be a weapon which he could use with advantage in bludgeoning reluctant Governments ${ }^{53}$.

De fait, le journal attribuait la défaite des libéraux dans les circonscriptions de Gilbert Plains, Dauphin, Beautiful Plains, Lakeside, Rockwood, Emerson, Dufferin, Manitou et Brandon, à une cabale Roblin-Langevin-nationaliste-ruthène. C'était là une coterie dont il fallait se défier. Sur ces entrefaits, La Liberté, porte-parole de $\mathrm{M}^{\mathrm{gr}}$ Langevin, lui lança ces ripostes :

Cela demeure sur l'estomac du Free Press que le gouvernement ait été maintenu au pouvoir par le vote des minorités. La maigreur de la majorité obtenue par le Gouvernement lui rend son échec encore plus pénible, plus cuisant qu'une défaite complète. Se serrer le ventre durant cinq autres années parce que les minorités ont donné leur vote contre ceux qui les menaçaient, quelle déception et que de colères $\mathrm{y}$ trouvent leur source ! 54

$52 \quad M F P$, le 11 février 1914.

53 Idem..

54 La Liberté, le 4 août, 1914. 
En 1915, les aubains étaient devenus aux yeux du Free Press une véritable menace pour la sécurité du Canada, ce qui poussait ce quotidien à exiger de plus en plus que tout Canadien soit sans attaches antérieures d'aucune sorte ${ }^{55}$. Puis il donnait un avertissement aux chefs religieux français que, si on persistait à réclamer pour les autres nationalités la jouissance des mêmes droits qu'eux ils devraient renoncer à toute chance d'obtenir des concessions ${ }^{56}$. Langevin était disparu entretemps, mais c'est toujours à lui que l'on reprochait d'avoir fait cause commune avec les minorités et d'être coupable d'avoir inondé la Province d'instituteurs qui savaient tout, sauf l'anglais. C'était lui qui avait déjọé les tenants de l'unilinguisme.

1916 : On a beaucoup parlé de cette abrogation. Dû au scandale de la construction du Palais législatif, dû à cette guerre qui avait attisé des susceptibilités nationalistes qui confinaient à l'hystérie, dû à une ferveur nouvelle envers l'Empire britannique et à la crainte de la "balkanisation » de l'Ouest-canadien, dû aux libéraux sous la direction de Tobias Crawford Norris qui avaient conquis le pouvoir de haute main, l'état d'esprit du moment fit que l'on voulut se défaire du bilinguisme au Manitoba, en tout et partout. La chauvinisme anglo-saxon s'en prit à tous les centraux qui menaçaient de supplanter le «Canadian way of life ». Il s'en suivit que les écoles publiques devaient de toute nécessité devenir surtout des agents d'assimilation et des inculcateurs de valeurs sociales reconnues ${ }^{57}$.

Des Franco-manitobains se crurent seuls visés quand on abrogea l'article 258 de la loi des écoles publiques. Mais il y avait autre chose et c'est par ricochet qu'ils étaient atteints. C'était le résultat d'une situation dont ils n'étaient pas directement responsables. En effet, le Free Press avait démontré de temps à autre une certaine compréhension du fait français au Manitoba :

The insistence of the French upon their language and their institutions is natural and understandable. Canada is their country as it is the country of the English-speaking Canadians. It was their country, indeed first; and the fact of conquest

$55 \quad M F P$, le 20 novembre 1915.

56 MFP, le 25 octobre 1915.

57 Maurice K. MotT, 'Foreign Peril' : Nativism in Winnipeg, 19161923, University of Manitoba, (Thèse de maîtrise, 1970), 73. 
is not so important as many think. So far as the French are concerned, it is necessary to bear in mind the fact that they occupy a special position, and are entitled to exceptional consideration. That they should cherish their sense of nationality is inevitable ${ }^{58}$.

L'affaire était donc très compliquée et la question pas facile à trancher. P.-A. Talbot, député libéral à la Législature provinciale, exposait clairement la situation dans une lettre à Wilfrid Laurier en janvier 1916 :

Ce qu'il y a de malheureux et qui complique notre position est que les Allemands, les Polonais, les Ruthènes ont depuis 15 ans pris avantage de la clause... et qu'aujourd'hui, sur les 436 écoles bilingues que nous avons dans la province, 310 sont soit allemandes, ruthènes ou polonaises et que tous les jours, 53 nouvelles demandes se font toujours se basant sur les privilèges que leur donne le règlement Laurier-Greenway pour demander des écoles bilingues et où l'anglais est très peu enseigné, vu la difficulté de trouver des instituteurs connaissant soit l'allemand et l'anglais ou soit le polonais etc... Aussi il est assez difficile de blâmer les Anglais de vouloir cesser cet état de choses, mais comment faire sans affecter les canadiens-français... S'il était possible de retrancher du règlement Laurier-Greenway " or any other language », je crois que cela serait la solution mais on prétend, et assez justement que de laisser immoler les allemands, les polonais et les ruthènes maintenant, cela affaiblira notre cause, et nous redeviendrons une quantité négligeable car nul doute que le but de Mgr Langevin en encourageant l'état de choses qui existe aujourd'hui était de se renforcer afin de pouvoir réouvrir la question des écoles séparées un jour ou l'autre ${ }^{59}$.

Laurier espérait quand même qu'une solution était possible :

Je suis absolument sûr d'après les conversations que j'ai eues avec Norris et Brown, que le gouvernement du Manitoba est sympathique aux Canadiens-français et ne demande pas mieux que de leur conserver les privilèges qu'ils ont en vertu du règlement Laurier-Greenway. Mais la situation est compliquée... par suite des abus qui ont été commis... C'est lui

58 MFP, le 29 juillet 1914.

59 Archives publiques du Canada (désormais APC) Fonds Laurier, P.-A. Talbot à Wilfrid Laurier, le 29 janvier 1916. 
(Langevin) qui a poussé les Ruthènes à demander des écoles bilingues 60 .

N'empêche qu'en mars 1916, le gouvernement Norris avait décidé de ne pas accorder de statut spécial à la langue française. Une lettre de J.W. Dafœ, écrite en avril 1916, donnait une explication partielle de cette décision:

There was a very general disposition on the part of those charged with dealing with the situation to retain upon the Statute Book certain privileges for the French... If it was found politically impossible to do this, the responsibility rests upon the French people of this Province and their leaders... in particular the clergy... there was a conspiracy, the moving spirit of which was the head of the Roman Catholic church in this diocese, to reduce the teaching of English to a minimum. Further, the influence which turned the supposed bilingual schools into French clerical schools in defiance of the law, and, in contempt of the agreement of 1897... deliberately instigated the Ruthenians and Polish sections of the community to claim, under the school law, the rights which it was the intention in 1897 to limit to the French alone... The agitation against bilingualism in Manitoba was directed primarily against the System of Slavic schools... If the people, in wiping them out, did not distinguish between them and the French schools... the French. the Polish, and the Ruthenians having made common cause, invited and received identical treatment 61 .

L'archevêque de Saint-Boniface était en bonne partie responsable de cet état de choses. Il avait suscité chez les Anglo-saxons une forte opposition au français, en associant la cause française avec celles des minorités étrangères. De fait, son empire catholique ne pouvait se réaliser qu'au moyen des écoles bilingues. Pas surprenant que les Anglo-saxons aient vu en cet homme, bien en vue chez les Canadiens français, un obstacle à l'accomplissement de leur destinée au Manitoba. Il avait, par le passé, encouragé les Allemands, les Polonais et les Ruthènes à ne pas abandonner leur langue maternelle; il s'était opposé à l'assimilation; et il avait contribué à l'accroissement du «foreign peril ». Pendant vingt ans, il avait, selon eux, mêlé la cause française à celle des immigrants de l'Europe

60 APC, Fonds Laurier, Wilfrid Laurier à P.-A. Talbot, le 2 février 1916.

61 University of Manitoba, Fonds Defœ, John W. Dafœ à Thomas Côté, le 6 avril 1916. 
centrale en vue d'obtenir, grâce à la force du nombre, un règlement de la question des écoles à sa convenance. Il avait assuré au gouvernement Roblin un grand nombre de votes, ce qui avait permis à celui-ci, de protéger les droits des minorités non-anglaises. C'est ainsi que les Canadiens français se trouvaient impliqués. À l'invitation de leur archevêque, ils avaient contribué à maintenir au pouvoir un gouvernement corrompu qui pendant longtemps avait permis la «balkanisation » au Manitoba. Aussi, pour s'être laissés entraîner par cet archevêque qui contrecarrait les vues anglo-saxones, ils s'étaient rendus sujet à des représailles.

On a beaucoup discuté de l'attitude de $\mathrm{M}^{\mathrm{gr}}$ Langevin. Certains l'ont approuvé hautement, de même que son comportement altier et ses ultimatums outranciers. D'autres prétendirent qu'il aurait fait mieux d'user d'un peu de diplomatie plutôt que de toujours batailler si éloquemment que ce fût. Quelque parti que l'on prenne, il faut reconnaître qu'il fut un personnage de premier plan et même un personnage de tragédie. Ce qui faisait sa force faisait aussi sa faiblesse. Une certitude doctrinale de son droit absolu, jointe à une détermination de réussir, causa la ruine d'une cause qui lui était chère. Il ne doutait pas de la justesse de ses objectifs, il n'admettait pas qu'ils fussent mal compris, ni qu'on les vit d'un mauvais oil ni que l'on prétendit qu'ils étaient à contretemps. S'il est difficile de sympathiser avec un tel homme il faut au moins tâcher de comprendre son œuvre: l'établissement de l'Église catholique universelle dans l'Ouest canadien.

Gilbert-Louis Comeault, B.A.(H)

Archiviste-adjoint aux Archives provinciales du Manitoba. 